\title{
Histone glutamine methylation afFACTing rDNA transcription
}

\author{
Cell Research (2014) 24:261-262. doi:10.1038/cr.2014.22; published online 14 February 2014
}

\begin{abstract}
Chemical modifications of histone proteins directly and indirectly affect chromatin structure and thereby contribute to the multilayered control of diverse DNA-based processes. A recent study in Nature enriches this list of enzyme-dependent posttranslational histone marks by $\mathrm{H} 2 \mathrm{~A}$ glutamine methylation that appears to be dedicated to only one specific cellular process, the regulation of nucleolar rDNA transcription.
\end{abstract}

Decades of research have highlighted the importance of posttranslational protein modifications as means to regulate diverse cellular processes, such as signal transduction pathways, metabolic functions and gene expression. Histones, the proteinaceous constituents of the packaged eukaryotic genetic information called chromatin, are decorated with a vast amount of diverse posttranslational modifications (PTMs) that differ in their chemical nature, residue location and, ultimately, their biochemical effects on DNA-based processes [1]. Lysine acetylation, lysine and arginine methylation, as well as serine and threonine phosphorylation are so far the best studied histone marks among many others and help to locally manipulate DNA accessibility of the chromatin structure to control gene regulation, DNA repair, mitosis and apoptosis.

Now, a recent study published in Nature by the group of Tony Kouzarides extends this already enormous list of histone PTMs with an additional, unexpected and evolutionarily conserved one that, surprisingly, operates exclusively in the nucleolus and is dedicated to a specific cellular process, rDNA transcription and ribosome biogenesis [2].
Tessarz et al. [2] identified glutamine methylation on canonical human histone H2A at position 104 (Q104, and Q105 in yeast) by mass spectrometry. Glutamine methylation has so far only been found on translation termination factors and ribosomal proteins [3]. In accordance, they showed with a specific H2A glutamine methylation (H2AQme)-specific antibody that this novel histone PTM is exclusively restricted to the nucleolus. This is a surprising finding, since all known histone PTMs thus far are found throughout the nucleus, but not restricted to such a specialized subnuclear area.

To unravel the functional implication of this novel mark, they first set out to identify the responsible enzyme. After initial failure of many candidate approaches they finally used an extremely laborious unbiased "fishing" experiment and succeeded in identifying Nop1, a known yeast rRNA methyltransferase [4] and its human homolog fibrillarin [5], as the H2AQ104/105-specific methyltransferase. This enzyme is part of the RNA polymerase I (RNAPI) complex that facilitates transcription of nucleolar rRNA genes needed for ribosome biosynthesis, possibly explaining the exclusive nucleolar presence of $\mathrm{H} 2 \mathrm{AQme}$. Is $\mathrm{H} 2 \mathrm{~A}$ glutamine methylation indeed correlated with the process of rDNA transcription? To answer this question, Tessarz et al. performed ChIP-seq experiments in a genetically manipulated yeast strain in which $80 \%$ of all rDNA repeats are deleted and the remaining 20 copies are actively transcribed and almost devoid of nucleosomes [6]. When analyzing the entire yeast genome, they found an exclusive enrichment of H2AQ105me over the 35S rDNA transcription units on chromosome 12 that depends on RNAPI activity, indicating that active transcription is required for $\mathrm{H} 2 \mathrm{~A}$ glutamine methylation to be established. What functional implication does H2AQ105me have for the process of rDNA transcription? One important protein complex that is required for the efficient passage of polymerases through chromatin is the "facilitator of chromatin transcription" FACT, which has histone chaperone and nucleosome destabilizing activities [7]. Tessarz et al. wondered whether glutamine methylation could impact FACT association with chromatin or its remodeling functions. With another elegant unbiased approach, this time using a randomized 12-residue peptide phage library in an interaction screen, the group of Kouzarides identified a consensus sequence spanning H2AQ105 as FACT-interaction domain, thereby confirming a previous report [8]. Furthermore, peptide pull-down experiments demonstrated that methylation of H2AQ or the exchange of this residue with alanine significantly reduced FACT binding, suggesting that the FACT-H2A interaction is disrupted by glutamine methylation. The functional consequence was investigated using both a URA3 reporter-based assay and a run-on experiment to determine the RNAPI transcription rate in a H2AQ105A mutant yeast strain that phenocopies H2AQ105me in terms of FACT binding. This mutant showed increased transcription rates, an effect also observed in cells with decreased FACT complex protein level. Furthermore, the authors also showed that cells with decreased FACT level or the H2AQ105A 


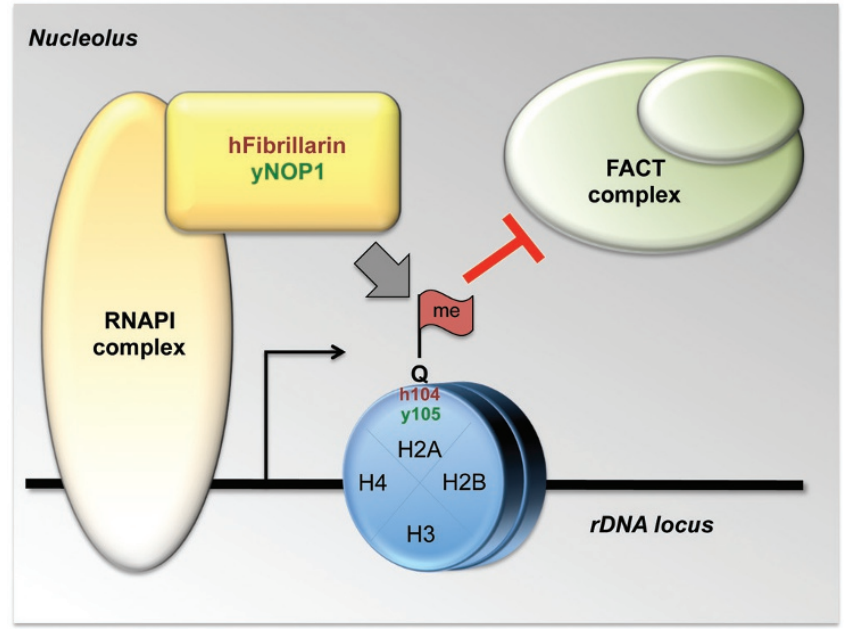

Figure $1 \mathrm{H} 2 \mathrm{~A}$ glutamine $(\mathrm{Q})$ methylation by human (h)Fibrillarin or yeast (y)NOP1 methyltransferase at the rDNA locus impedes FACT binding, providing a novel epigenetic link between RNA polymerase I (RNAPI) transcription and ribosome biogenesis.

mutant have a pronounced reduction in H2A chromatin incorporation. Finally, they overexpressed Nop1 and demonstrated that the resulting increased methylation of H2AQ105 correlates with a decrease in FACT occupancy at the rDNA locus. Together, these data suggest that reduced FACT activity or glutamine methylation of $\mathrm{H} 2 \mathrm{~A}$ can both lead to a more open chromatin structure and reduced $\mathrm{H} 2 \mathrm{~A}$ chromatin incorporation, thus providing the basis for a higher rDNA transcription rate.

The above findings by Tessarz et al. are highly interesting as they open new perspectives on RNAPI transcription regulation, provide new links to ribosome biogenesis, assign a new function to a long acquainted enzyme (fibrillarin) and open up many new questions for future studies. In particular, how can one explain the finding that reduced FACT-chromatin binding due to $\mathrm{H} 2 \mathrm{~A}$ glutamine methylation leads to increased rDNA transcription rates, while FACT has been show to be required for the efficient passage of, at least, RNAPII through chromatin [9]? The group of Kouzarides proposes a model in which FACT depletes most of $\mathrm{H} 2 \mathrm{~A}$ from rDNA chromatin, resulting in low nucleosome occupancy with a residual reloading of glutamine methylated $\mathrm{H} 2 \mathrm{~A}$ by the chaperone NAP1. At this point, more detailed mechanistic and possibly kinetic data are required to unravel the precise functional relationship between FACT and H2AQme in rDNA transcription.

Finally, considering these fascinating new insights in rDNA regulation, it might be worthwhile to have a closer look at a potential role of histone glutamine methylation in tumorigenesis. It is known that the tumor suppressor p53 represses fibrillarin and that p53- inactivated cancer cells have high levels of this methyltransferase and rRNA methylation [10]. Thus, is it possible that increased histone glutamine methylation occurs in cancer and, thereby, contributes to the malignant cell transformation by enhancing rDNA transcription? Only future studies will tell!

\section{Heinrich Leonhardt ${ }^{1}$, Sandra B Hake ${ }^{2}$}

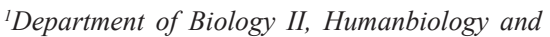
Bioimaging, Center for Integrated Protein Science (CIPSM), Ludwig Maximilians University Munich, 82152 Planegg-Martinsried, Germany; ${ }^{2}$ Molecular Biology Unit, Adolf-Butenandt-Institute, Center for Integrated Protein Science Munich (CIPSM), Ludwig Maximilians University Munich, 80336 Munich, Germany

Correspondence: Heinrich Leonhardta ${ }^{\mathrm{a}}$, Sandra B Hake $^{\mathrm{b}}$

aTel: +49 892180 74232; Fax: +49 892180

74236

E-mail: h.leonhardt@Imu.de

'Tel: +49 892180 75435; Fax: +49 892180

75425

E-mail: sandra.hake@med.uni-muenchen.de

\section{References}

1 Strahl BD, Allis CD. Nature 2000; 403: 4145.

2 Tessarz P, Santos-Rosa H, Robson SC, et al. Nature 2014; 505:564-568.

3 Polevoda B, Sherman F. Mol Microbiol 2007; 65: 590-606.

4 Tollervey D, Lehtonen H, Jansen R, et al. Cell 1993; 72: 443-457.

5 Jansen RP, Hurt EC, Kern H, et al. J Cell Biol 1991; 113: 715-729.

6 Ide S, Miyazaki T, Maki H, Kobayashi T. Science 2010; 327: 693-696.

7 Formosa T. Biochim Biophys Acta 2012; 1819: 247-255.

8 VanDemark AP, Xin H, McCullough L, et al. J Biol Chem 2008; 283: 5058-5068.

9 Belotserkovskaya R, Saunders A, Lis JT, et al. Biochim Biophys Acta 2004; 1677: 8799.

10 Marcel V, Ghayad SE, Belin S, et al. Cancer Cell 2013; 24: 318-330. 